\title{
Recension d'ouvrage
}

\author{
Claude Juin, Des soldats tortionnaires. Guerre \\ d'Algérie: des jeunes gens ordinaires confrontés à \\ l'intolérable, Paris : Robert Laffont, 2012, 363 p.
}

\author{
Brieg Capitaine \\ (Professeur adjoint au département de sociologie \\ et d'anthropologie de l'Université d'Ottawa)
}

Avant d'être un témoignage sur la cruauté dont a pu faire preuve l'armée française pendant «les évènements d'Algérie », l'ouvrage de Claude Juin, adapté d'une thèse de doctorat soutenue à l'EHESS en 2011, pose une question sociologique importante. Comment de jeunes gens appelés sous les drapeaux - qualifiés d' «ordinaires » par l'auteur - sont-ils devenus des soldats tortionnaires?

Dans cet ouvrage, la position de Claude Juin est triple : témoin, acteur des mouvements de décolonisation et chercheur. L'auteur s'appuie ainsi d'une part sur ses propres notes et observations de la vie au cantonnement, et d'autre part sur des témoignages d'anciens combattants qu'il a interrogés ou des extraits d'archives. Le lecteur circule ainsi en permanence entre le regard porté sur les autres et sur soi, entre objectivité et subjectivité. Cette position méthodologique peut surprendre, voire agacera très certainement certains orthodoxes de l'analyse sociologique. Elle sert pourtant brillamment l'analyse dans la mesure où elle attire l'attention du lecteur sur le statut du témoignage, de l'écriture comme forme de reconstruction de soi. Elle informe également par un effet de miroir sur le passage à la violence qui semble s'imposer à mesure que disparaît cette capacité de regard sur soi.

Dans la première partie, l'auteur précise d'emblée que ces jeunes soldats furent mobilisés non pas pour faire la guerre, mais pour mener sur le territoire algérien une «mission de pacification »- le terme de guerre ne fut reconnu officiellement qu'en 1999. Cette euphémisation langagière informe sur l'idéologie coloniale et impérialiste qui, malgré l'importance accordée aux droits humains, prévaut au sortir de la Seconde Guerre mondiale. Les jeunes appelés plongèrent ainsi dans le paradoxe d'un univers colonial où les intérêts de la patrie s'opposaient aux droits de l'homme et au principe d'autodétermination des peuples rappelé pourtant par Pierre Mendès France en 1954 alors même que des bateaux emplis de soldats - déclarés bons pour le service après trois jours de formation - traversaient la Méditerranée. Les nombreux témoignages sur lesquels s'appuie l'auteur rendent compte de la confusion qui frappait les jeunes appelés dès leur arrivée à Alger. La nervosité des soldats présents, grenades à la ceinture et la traversée d'Alger avec les fusils mitrailleurs bra- 
qués sur les civils entraient en dissonance avec la représentation de ce que devait être leur mission de pacification.

L'auteur, à travers les témoignages, montre comment la figure alors dominante de l'Arabe se fissure, comment les questionnements sur l'histoire se font plus forts, comment les incertitudes et les doutes s'immiscent dans la conscience des désormais soldats du Djebel. Ceux-ci mesurent les inégalités et les injustices qui structurent alors les rapports sociaux en Algérie. Ils font face aux exactions commises par leurs â̂nés, au racisme des colons, mais perdent aussi leurs premiers «copains » tués en opération. Dès l'arrivée au cantonnement, les appelés font l'expérience du racisme, de l'arbitraire. Le froid, la peur, l'ennui, l'envie de vengeance finissent de fissurer les consciences et marquent l'avènement du désordre. Selon l'auteur, étourdis par cette plongée soudaine dans la guerre meurtrière, de nombreux jeunes s'abandonnèrent au discours de la cellule d'action psychologique de l'armée ou à l'arbitraire de leur commandement. «Il n'appartenait pas au jeune soldat de démêler les paradoxes de l'action militaire et du pouvoir d'État. Sinon il perdait le sens de sa mission » note Claude Juin (p.298) qui rend compte dans cette première partie des effets d'une structure, des discours sur une génération de jeunes alors en pleine construction de soi.

Les descriptions faites par l'auteur dans la seconde partie sont bien éloignées de la franche camaraderie qui se dégage parfois des clichés de campagne militaire. L'ambiance "cannette de bière », les bagarres, les soûleries, l'arbitraire des ordres et la désobéissance décrits dans l'ouvrage tranchent avec l'idée de l'armée comme un corps solidaire, unifiée, où l'ordre se résume à la stricte correspondance entre rôle et statut. L'auteur qui instille plutôt le sentiment d'entrer dans un univers étrange, fait d'émotions, de rancœur et de pulsions meurtrières donne à penser l'institution militaire plongée dans le mensonge de la «pacification », comme un lieu de déstructuration, de destruction progressive de la conscience des jeunes à mesure que se prolonge leur expérience du conflit. La durée de mobilisation des soldats repoussée tous les deux mois achève d'instaurer une mauvaise ambiance générale. Les lettres envoyées en métropole se succèdent et décrivent souvent le «sale boulot» de soldat ou la «corvée de bois » qui désignait les exécutions sommaires d'Algériens soupçonnés d'appartenir au FLN. Toutefois, seule une minorité rapporta les exactions commises par leurs camarades, d'autres, de retour en métropole, dénoncèrent publiquement la torture. Ils furent sans cesse censurés, certains arrêtés et accusés d'être des ennemis de la patrie. L'Algérie française et l'idéologie coloniale restèrent, selon l'auteur, la norme de l'époque et le prisme central de l'analyse du conflit. L'exemple de Bernard, un jeune homme ordinaire en métropole et devenu véritable bourreau à la ferme Moll, est à ce titre particulièrement illustratif de la force de cette idéologie sur le passage à la violence. Mais, le portrait serré que l'auteur dresse de son «copain » éclaire surtout le lecteur sur le poids de la subjectivité dans le passage à la violence. La cruauté surgit ici de manière fulgurante et si les bourreaux trouvent 
dans l'idéologie coloniale une forme de légitimation de leurs actes, Claude Juin par ses descriptions et ses notes prises à chaud amène le lecteur à voir l'irruption de la violence comme un système subjectif formé par la peur, les refoulements, les incertitudes, l'affect et la sensibilité. En même temps, la place de la subjectivité donnée par l'auteur dans ce processus de cruauté est accentuée par les notes prises par lui ; ce regard sur soi semblant ainsi le préserver de la désubjectivation. Néanmoins, le lecteur ne trouvera pas d'explication causale univoque sur les ressorts de la violence, et parfois, au risque de se perdre, l'auteur privilégie la circulation entre le monde des institutions et celui des individus.

La troisième partie se penche sur le retour de la guerre et plus particulièrement sur la place de la mémoire dans l'engagement ou le silence de cette jeunesse une fois démobilisée. Certains témoignent, écrivent, parlent pour rendre compte de leur expérience et ainsi rétablir la vérité historique face à l'hypocrisie du pouvoir politique. Mais ils ne furent qu'une minorité. Claude Juin qui milita au sein de ces mouvements note que ceux qui ont brisé le silence, qui ont témoigné des violences observées ou qui s'y sont parfois violemment opposés sur le terrain «avaient acquis une conscience sociale et politique plus élaborée que la plupart des autres jeunes, [ils avaient conscience] du sens de la lutte engagée par les combattants algériens » (p.70). Le militantisme apparut ainsi comme une forme d'engagement dans un collectif, comme un moyen de refouler l'isolement dont semblaient souffrir les anciens soldats du djebel prisonniers de leur mémoire. Il s'agissait également, selon l'auteur, de donner un sens à sa vie, de rechercher la certitude du combat idéologique contre la colonisation après avoir été aussi durement questionné dans son rapport au politique ou à l'histoire. Ils ne furent qu'une minorité à suivre cette voie, car ce qui caractérise plutôt le retour de la guerre est le silence.

Pourquoi la plupart des jeunes démobilisés choisirent-ils de se taire ? «Pourtant, on aurait voulu être entendus, mais ce n'était pas possible. On se moquait plutôt de nous, ceux qui avaient fait l'Algérie » (p.281) raconte un vétéran. Officiellement, il n'y avait pas eu de guerre. Ils n'étaient donc pas des anciens combattants aux yeux de la société française. Abandonnés par l'histoire nationale, ils ne trouvèrent aucune écoute auprès de la société. Le silence des anciens fut également entretenu par euxmêmes. Ils cherchèrent à recouvrer leur place et firent semblant d'oublier, après avoir été acteurs ou témoins de la cruauté humaine. L'oubli pourtant ne favorisa ni la reconstruction d'une démocratie juste et respectueuse des droits humains, ni la compréhension par les anciens du «djebel» des raisons qui avaient pu leur faire commettre ces violences extrêmes. Aujourd'hui, les anciens d'Algérie parlent et témoignent. Mais les mémoires se libèrent dans la souffrance et illustrent la difficile et lente reconstruction identitaire. L'auteur restitue de manière très fine l'atmosphère et le contenu des témoignages qu'il a récemment récoltés auprès d'anciens tortionnaires : «La voix est hésitante, les mots sont comme une délivrance d'un vilain songe » (p.279). Bernard raconte : «J'ai fini par parler, mais ce jour-là, je n'ai fait 
que pleurer parce que les images revenaient » (p.230). «J'ai hésité longtemps, mais ça suffit ce silence. Dire les choses aidera peut-être les jeunes générations à réfléchir à la question de la haine et de la guerre. Et puis de toute façon, j'ai besoin que ça sorte, car je me sens un salaud. C'est un soulagement pour moi de pouvoir dire tout cela » (p.230-231) témoigne également André. Les traumatismes psychiques pour faits de guerre, dont souffrent encore aujourd'hui des anciens soldats de la guerre ont poussé quelques-uns d'entre eux au suicide. Ces traumatismes sont peu reconnus comme handicap et ne font pas l'objet de rentes spécifiques. Néanmoins, l'auteur note que cette situation évolue avec la création au sein de l'armée française de la cellule d'intervention et de soutien psychologique de l'armée de terre.

À première vue, l'ouvrage de Claude Juin peut choquer certains anciens soldats qui ont conservé le silence ou apparaître par ses révélations comme faisant recours au sensationnalisme. Il constitue néanmoins un travail de sociologie clinique remarquable pour toute cette génération de jeunes appelés sous les drapeaux. Les logiques coloniales implacables, l'idéologie impérialiste, les mensonges du pouvoir politique, l'absence de préparation, le désordre, l'arbitraire, la faiblesse du commandement expliquent comment ces jeunes livrés à eux-mêmes se sont transformés en bourreaux. Claude Juin montre alors comment l'écriture ou le témoignage est une voie féconde dans les processus de reconstruction d'une démocratie qui a bafoué au nom de ses intérêts particuliers, les valeurs de justice sur lesquelles elle repose. La reconnaissance officielle de la guerre d'Algérie, du statut d'ancien combattant et de manière plus générale des souffrances post-traumatiques qui affectent de nombreux soldats apparaissent ainsi comme autant d'avancées sociales et culturelles consécutives à ce mouvement de libération des mémoires et au projet de construction d'une démocratie plus proche de son idéal de justice 